\title{
WDM Transmission Using Dispersion Compensation in Optical Transmission Links with Nonuniform Residual Dispersion per Span
}

\author{
Seong-Real Lee, Member, KIICE
}

\begin{abstract}
The possibility of implementing nonuniform residual dispersion per span (RDPS) in optical links with net residual dispersion (NRD) controlled by precompensation and postcompensation for 960 Gbps WDM transmissions is studied and discussed. The fiber optic communication links investigated in this paper consist of inline dispersion management (DM) for each fiber spans and optical phase conjugator (OPC) at mid-way of total transmission length in order to compensate for WDM signal distortions due to group velocity dispersion (GVD) and nonlinearities. It is confirmed that the effect of nonuniform RDPS distribution on system performance is not significant. It is also confirmed that the optimal NRD is obtained to be one of two values of +10 ps/nm or $-10 \mathrm{ps} / \mathrm{nm}$, which depend on the deciding of NRD controlled by precompensation or postcompensation, and the exact RDPS configurations. The effective NRD ranges resulting eye opening penalty (EOP) below $1 \mathrm{~dB}$ are independent on the exact RDPS distribution for relative low launch power. Therefore, results show the possibility of implementing the flexible optical links to expand network construction for WDM transmission of high bit-rate capability.
\end{abstract}

Index Terms - Dispersion management, Optical phase conjugator, Residual dispersion per span (RDPS), Nonuniform RDPS configuration, Net residual dispersion (NRD), pre(post)compensation, group velocity dispersion, self phase modulation

\section{INTRODUCTION}

FIBER optics communication has been growing as a novel technology over the past decades. Especially, optical wavelength-division multiplexed (WDM) system is currently very much preferred because of its high bit rate capability and of the successful implementation of optical amplifiers such as an erbium-doped fiber amplifier (EDFA). The demand for transmission over the global telecommunication network is increasing at faster rate, and fiber optics has the potential to meet the demand. However, fiber dispersion and nonlinearity degrade propagating pulses causing communication errors. In long haul communication systems, dispersion management (DM) is used to minimize the pulse broadening. However, the output pulse shape also depends upon the signal power level through nonlinear effects, such as four-wave mixing

Manuscript received July 13, 2011; revised September 9, 2011; accepted September 17, 2011

Seong-Real Lee is with the Division of Marine Electronic and Communication Eng., Mokpo National Maritime University, Joennam, 530-729, Korea (Email: reallee@mmu.ac.kr)
(FWM), self- phase modulation (SPM) and cross-phase modulation (XPM), cause performance degradation of the optical WDM system.

In the absence of nonlinearity, the effects of group velocity dispersion (GVD) can be compensated, by using dispersion compensating fiber (DCF) [1], to recover original signal accurately. However this is not the case when nonlinear effects cannot be neglected. SPM is the dominant nonlinear phenomenon that limits the performance of single channel light wave systems [1],[2]. SPM gives rise to an intensity dependent nonlinear phase shift which introduces chirp in the propagating signal and hence causes spectral broadening. In long haul communication link, the SPM effects accumulate due to inline amplifiers which are used periodically for loss compensation.

In the presence of fiber GVD and SPM, the pulse spread is sensitive to dispersion map as well as the values of launch power. Hence for the designing of optical fiber communication links, it is necessary to consider the combined effects of GVD and SPM on pulse propagation along the fiber link and to explore the optimum choice for the amount of dispersion compensation at various locations to minimize pulse degradation. Furthermore, it is also necessary to consider fiber spans having different residual dispersion per span (RDPS) with each other, for designing of flexible optical fiber communication links.

However, most of the previous studies on dispersion map were focused on the fixed RDPS for every fiber span. This means that, if such a dispersion map is used, the length of fiber span including DCF have to be fixed, hence, the implementation of reconfigurable WDM optical mesh networks is very difficult. This complicates the system control and increases the system cost.

Optical phase conjugation is another effective technique to reduce GVD and nonlinear impairment mainly due to SPM. The compensation for signal impairment in this technique is theoretically possible through the use of optical phase conjugator (OPC) in the middle of total transmission length. But, the effective suppression of nonlinear impairment is not practically obtained in WDM transmission systems, because nonlinearity cancellation requires a perfectly symmetrical distribution of power and local dispersion with respect to OPC position. Due to the presence of fiber attenuation, this condition cannot be satisfied in real links [3]. Author had shown the implementation possibility of WDM transmission system with good receive performance by applying DM and OPC into optical links through the previous studies [4]-[6]. 
In this paper, a modified periodic dispersion map with different RDPS for each fiber span is proposed for 960 Gbps ( $=24$ channels $\times 40 \mathrm{Gbps}) \mathrm{WDM}$ systems with OPC at mid-way of total transmission length. The detail schemes of dispersion map considered in this research are two configurations as following; first, RDPS from transmitter (Tx) to OPC are increased, meanwhile, RDPS from OPC to receiver $(\mathrm{Rx})$ are decreased as transmission distance is increased, this configuration is called to 'ascending-descending RDPS configuration', and second, RDPS from transmitter (Tx) to OPC are decreased, but RDPS from OPC to receiver ( $\mathrm{Rx})$ are increased as transmission distance is increased, this configuration is called to 'descending-ascending RDPS configuration'.

In this paper, optimal net residual dispersion (NRD) of links designed by ascending-descending RDPS configuration and descending-ascending RDPS configuration will be induced, respectively. Also, effective NRD ranges in each case will be induced as a function of values of launch power of WDM channels through a numerical study of assessing eye opening penalty (EOP) of worst channel among 24 WDM channels.

\section{CONFIGURATIONS OF OPTICAL LINKS AND WDM SYSTEM}

Optical transmission link configuration including WDM Tx section and Rx section investigated in this research is shown in Fig. 1. Total transmission links consists of 12 spans, in which including SMF with $80 \mathrm{~km}$ length, i.e., $l_{S M F, n}=80 \mathrm{~km}$ (where, $n$ is span number) and DCF with variable length depending on RDPS. SMF was characterized by the attenuation coefficient $\alpha_{S M F}=0.2$ $\mathrm{dB} / \mathrm{km}$, dispersion coefficient $D_{S M F}=17 \mathrm{ps} / \mathrm{nm} / \mathrm{km}$, and the nonlinear coefficient $\gamma_{S M F}=1.41 \mathrm{~W}^{-1} \mathrm{~km}^{-1}$ at $1,550 \mathrm{~nm}$. Thus, accumulated dispersion in each SMF is $1,360 \mathrm{ps} / \mathrm{nm}$ $(=17 \mathrm{ps} / \mathrm{nm} / \mathrm{km} \times 80 \mathrm{~km})$.

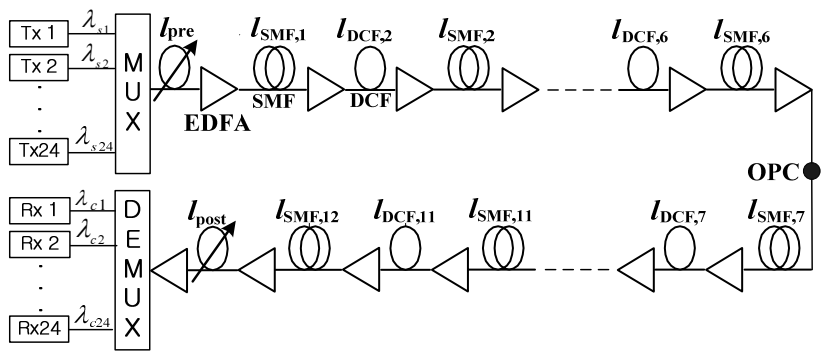

Fig. 1. Configuration of optical links and WDM transmission system.

In this research, the averaged RDPS are assumed to be equal value of $150 \mathrm{ps} / \mathrm{nm}$ in both transmission sections from Tx to OPC and from OPC to Rx, except first fiber span and last fiber section. If RDPS is selected to be the fixed $150 \mathrm{ps} / \mathrm{nm}$, as plotted in Fig. 2(a), the length of DCF, i.e., $l_{D C F, n}(\mathrm{n}=2,3,4, \cdots, 11)$ has to be $12.1 \mathrm{~km}$ at each fiber span, except first and last fiber section. This case is called to the 'uniform RDPS distribution' in this paper.

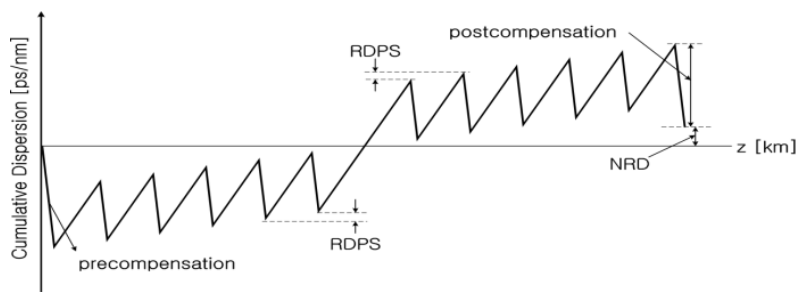

(a) uniform RDPS distribution

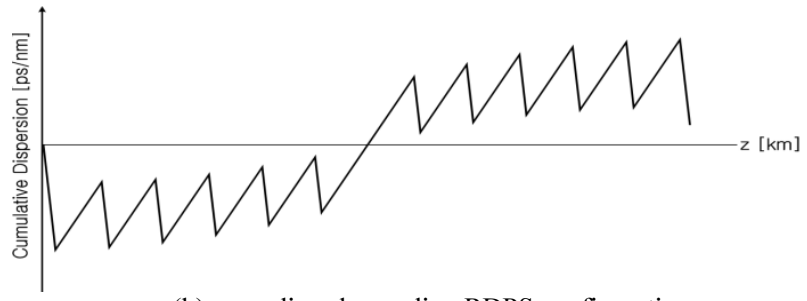

(b) ascending-descending RDPS configuration

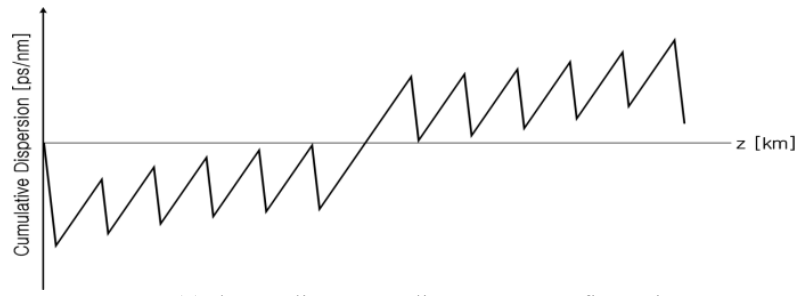

(c) descending-ascending RDPS configuration

Fig. 2. Dispersion maps.

TABLE I

RDPS AND $L D C F$

\begin{tabular}{|c|c|c|c|c|}
\hline \multirow{2}{*}{$\begin{array}{c}\text { Span } \\
\text { Number }\end{array}$} & \multicolumn{2}{|c|}{ Ascending-descending } & \multicolumn{2}{|c|}{ Descending -ascending } \\
\hline & RDPS & $l_{D C F}$ & RDPS & $l_{D C F}$ \\
\hline 1 & \multicolumn{2}{|c|}{ precompensation } & \multicolumn{2}{|c|}{ precompensation } \\
\hline 2 & 50 & 13.1 & 250 & 11.1 \\
\hline 3 & 100 & 12.6 & 200 & 11.6 \\
\hline 4 & 150 & 12.1 & 150 & 12.1 \\
\hline 5 & 200 & 11.6 & 100 & 12.6 \\
\hline 6 & 250 & 11.1 & 50 & 13.1 \\
\hline 7 & 250 & 11.1 & 50 & 13.1 \\
\hline 8 & 200 & 11.6 & 100 & 12.6 \\
\hline 9 & 150 & 12.1 & 150 & 12.1 \\
\hline 10 & 100 & 12.6 & 200 & 11.6 \\
\hline 11 & 50 & 13.1 & 250 & 11.1 \\
\hline 12 & \multicolumn{2}{|c|}{ nsation } & \multicolumn{2}{|c|}{ postcompensation } \\
\hline
\end{tabular}

The RDPS for ascending-descending configuration, as plotted in Fig. 2(b), is designed as following; RDPS of second span is to be $50 \mathrm{ps} / \mathrm{nm}$ and RDPS of the rest span up to $6^{\text {th }}$ span are increased by $50 \mathrm{ps} / \mathrm{nm}$ than previous span from $100 \mathrm{ps} / \mathrm{nm}$ to $250 \mathrm{ps} / \mathrm{nm}$, meanwhile, RDPS of $7^{\text {th }}$ span is to be $250 \mathrm{ps} / \mathrm{nm}$ and RDPS of the rest span up to $11^{\text {th }}$ span are decreased by $50 \mathrm{ps} / \mathrm{nm}$ than previous span from $200 \mathrm{ps} / \mathrm{nm}$ to $50 \mathrm{ps} / \mathrm{nm}$, as summarized in Table 1 , 
under the condition of $150 \mathrm{ps} / \mathrm{nm}$ averaged RDPS. And, the RDPS for descending-ascending configuration, as plotted in Fig. 2(c), is designed to be the reverse distribution of ascending-descending configuration.

Dispersion coefficient of DCF of every span, $D_{\mathrm{DCF}}$ is assumed to be $-100 \mathrm{ps} / \mathrm{nm} / \mathrm{km}$ in all RDPS configurations. DCF was characterized by the attenuation coefficient $\alpha_{D C F}$ $=0.4 \mathrm{~dB} / \mathrm{km}$, and the nonlinear coefficient $\gamma_{D C F}=4.83 \mathrm{~W}^{-}$ ${ }^{1} \mathrm{~km}^{-1}$ at $1,550 \mathrm{~nm}$.

The NRD value will be varied by precompensation and postcompensation, which are determined by DCF lengths of first and last fiber section, i.e., $l_{\text {pre }}$ and $l_{\text {post }}$ plotted in Fig. 1 , respectively. The $l_{\text {pre }}$ and $l_{\text {post }}$ have to be decided to 21.1 $\mathrm{km}$ for $\mathrm{NRD}=0 \mathrm{ps} / \mathrm{nm}$, because total accumulated dispersion in one transmission section (from Tx to OPC or from OPC to $\mathrm{Rx}$ ) is $2,110 \mathrm{ps} / \mathrm{nm}$ due to the accumulated dispersion of $1,360 \mathrm{ps} / \mathrm{nm}$ in first or last SMF and sum of RDPS in five spans. In this paper, the NRD value is varied by precompensation and postcompensation from $-50 \mathrm{ps} / \mathrm{nm}$ to $+50 \mathrm{ps} / \mathrm{nm}$ by $10 \mathrm{ps} / \mathrm{nm}$ spacing, in order to induce optimal NRD for each RDPS configurations.

Tx illustrated in Fig. 1 is assumed to be distributed feedback laser diode (DFB-LD). The center wavelength of DFB-LD is assumed to be $1,550 \sim 1,568.4 \mathrm{~nm}$ by spacing $100 \mathrm{GHz}(0.8 \mathrm{~nm})$ based on ITU-T recommendation G.694.1. Hence, if each wavelength is allocated for one WDM channel, the considered total wavelength corresponds to 24-channel WDM transmission. DFB-LD is externally modulated by an independent 40 Gbps $128\left(=2^{7}\right)$ pseudo random bit sequence (PRBS). The modulation format from external optical modulator is assumed to be RZ. And output electric field of RZ format is assumed to be second-order super-Gaussian pulse with $10 \mathrm{~dB}$ extinction ratio (ER) and chirp-free. The duty cycle of RZ format is assumed to be 0.5 .

The nonlinear medium of OPC around mid-way of total transmission length is highly nonlinearity - dispersion shifted fiber (HNL-DSF). The parameters of OPC using HNL-DSF are as follows; loss of HNL-DSF $\alpha_{0}=0.61$ $\mathrm{dB} / \mathrm{km}$, nonlinear coefficient of HNL-DSF $\gamma_{0}=20.4 \mathrm{~W}^{-}$ ${ }^{1} \mathrm{~km}^{-1}$, length of HNL-DSF $z_{0}=0.75 \mathrm{~km}$, zero dispersion wavelength of HNL-DSF $\lambda_{0}=1,550 \mathrm{~nm}$, dispersion slope $d D_{0} / d \lambda=0.032 \mathrm{ps} / \mathrm{nm}^{2} / \mathrm{km}$, pump light power $P_{p}=18.5$ $\mathrm{dBm}$, and pump light wavelength $\lambda_{\mathrm{p}}=1549.75 \mathrm{~nm}$. The 3$\mathrm{dB}$ bandwidth of conversion efficiency $\eta$ of the OPC is obtained to be $48 \mathrm{~nm}(1526 \sim 1574 \mathrm{~nm})$. The signal wavelengths are converted to $1,549.5 \sim 1,528.5 \mathrm{~nm}$ (these are called to the conjugated wavelength) through OPC. Thus, allocated 24 signal wavelengths and these conjugated wavelengths are belongs within $3-\mathrm{dB}$ bandwidth of $\eta$.

The conjugated wavelength is sent into $\mathrm{Rx}$ of direct detection. Rx consists of the pre-amplifier of EDFA with 5 $\mathrm{dB}$ noise figure, the optical filter of $1 \mathrm{~nm}$ bandwidth, PIN diode, pulse shaping filter (Butterworth filter) and the decision circuit. The receiver bandwidth is assumed to be $0.65 \times$ bit-rate [7].

\section{SIMULATION RESULTS AND DISCUSSION}

Fig. 3 illustrates EOPs of worst channel among 24 WDM channels as a function of NRD controlled by precompensation and postcompensation, respectively, in optical transmission links of uniform RDPS distribution, ascending-descending RDPS configuration (signed by $\nearrow$ and $\searrow$ in legend of figure) and descending-ascending RDPS configuration (signed by $\searrow$ and $\nearrow$ ).

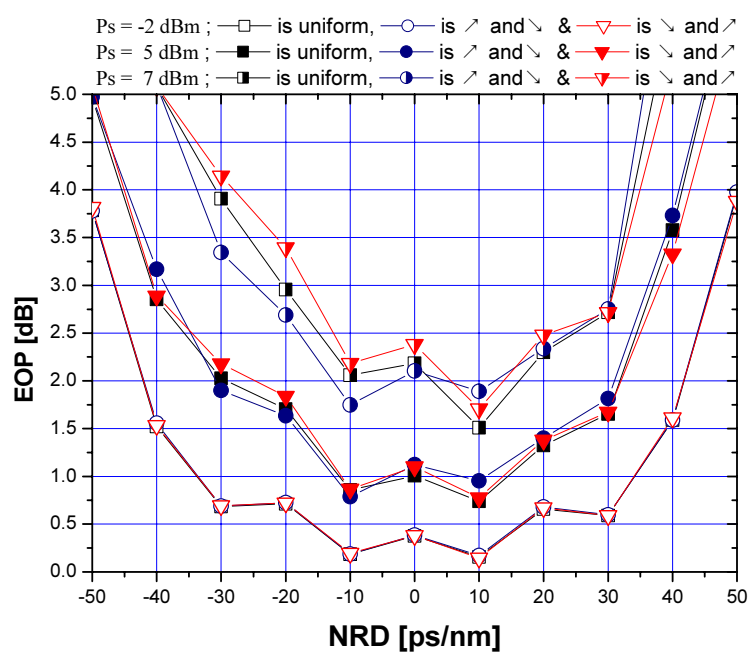

(a) precompensation

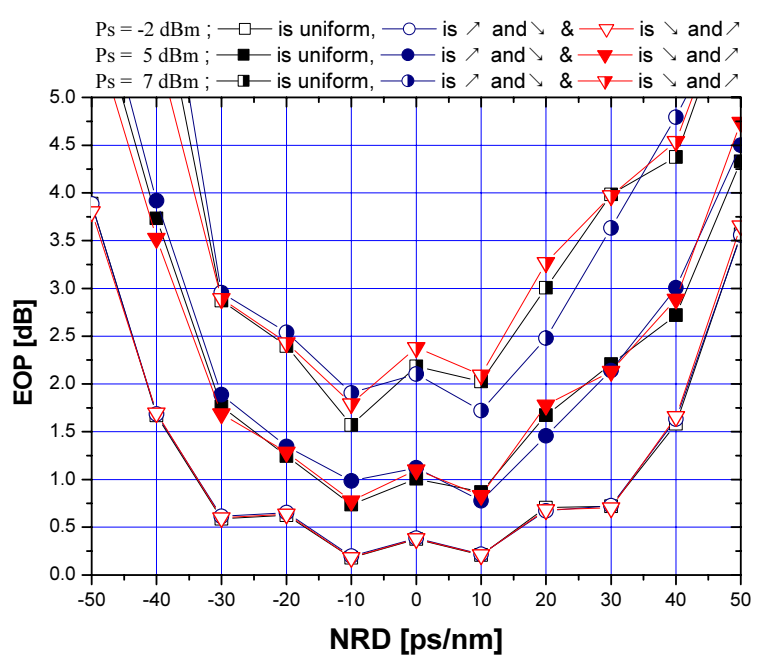

(b) postcompensation

Fig. 3. EOP as a function of NRD controlled by precompensation and postcompensation, respectively.

It is shown that EOPs depend on NRD and launch power $\left(P_{\mathrm{s}}\right)$ as well RDPS distribution. Especially, EOP becomes much sensitive under higher launch power. It is confirmed that the optimal NRDs are obtained to +10 $\mathrm{ps} / \mathrm{nm}$ and $-10 \mathrm{ps} / \mathrm{nm}$ in optical link controlled by precompensation and postcompensation, respectively, for uniform RDPS distribution and descending-ascending 
RDPS configurations. But, the optimal NRDs are obtained to $-10 \mathrm{ps} / \mathrm{nm}$ and $+10 \mathrm{ps} / \mathrm{nm}$ in optical link controlled by precompensation and postcompensation, respectively, for ascending-descending RDPS configurations.

In precompensation configuration, the width of optical pulses are compressed as these propagated through optical links of descending distribution of RDPS, thus initial temporal width of optical pulses in first fiber span are required to be appropriately broaden for the excellent compensation. The broadening of initial temporal width of optical pulses is accomplished by optimal NRD of +10 $\mathrm{ps} / \mathrm{nm}$ in this research. Meanwhile, because width of optical pulses are broadened as these propagated through optical links of ascending distribution of RDPS, initial temporal width of optical pulses in first fiber span are required to be appropriately compressed, and this condition is satisfyed by optimal NRD of $-10 \mathrm{ps} / \mathrm{nm}$ in this research. And, the opposite occurs for postcompensation configuration.

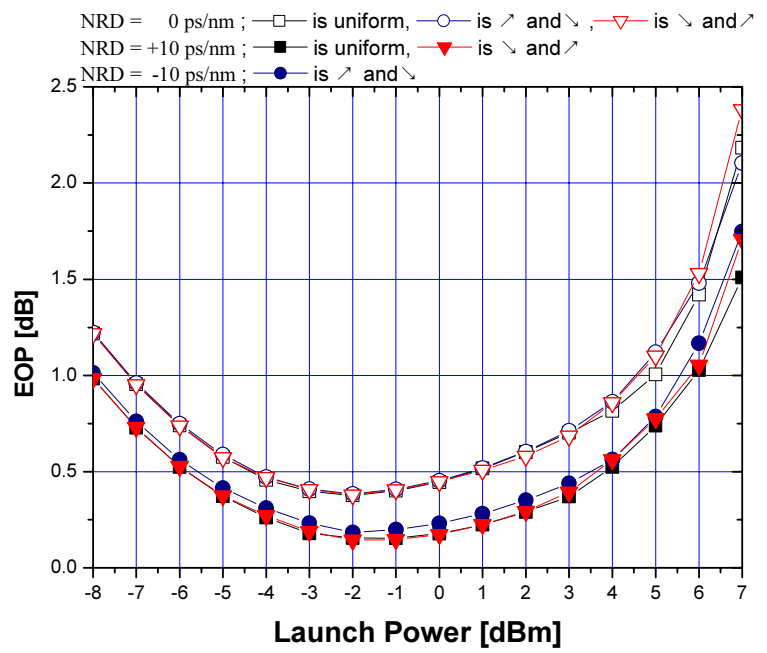

(a) precompensation

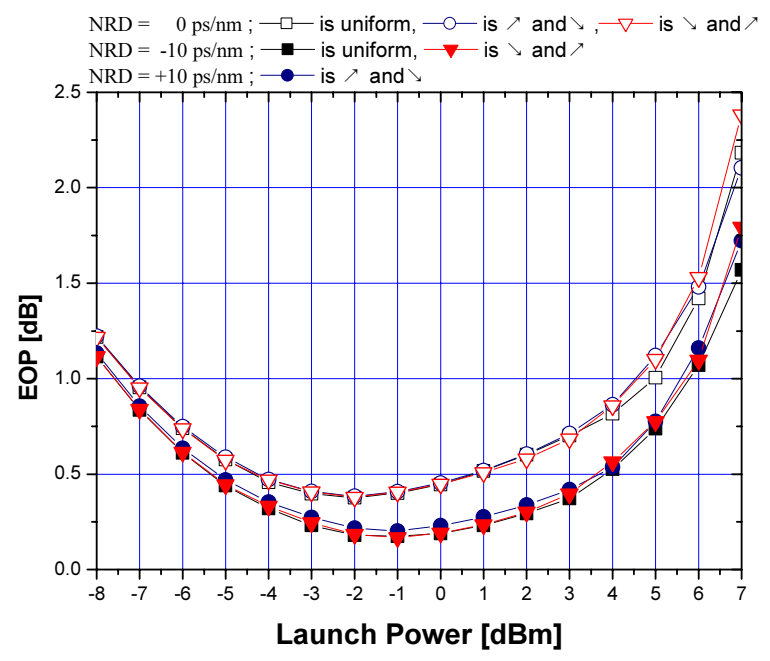

(b) postcompensation

Fig. 4. EOP of worst channel as a function of launch power.
Fig. 4 shows EOPs of worst channel as a function of launch power in optical links with NRD of $+10 \mathrm{ps} / \mathrm{nm}$ and $-10 \mathrm{ps} / \mathrm{nm}$, which are controlled by precompensation (shown in Fig. 4(a)) and by postcompensation (shown in Fig. 4(b)), respectively. These two results are also compared with the result in optical links with NRD of 0 $\mathrm{ps} / \mathrm{nm}$.

It is shown that, in both optical links controlled by precompensation and postcompensation, when NRD is decided to optimal value of $+10 \mathrm{ps} / \mathrm{nm}$ or $-10 \mathrm{ps} / \mathrm{nm}$ resulted from Fig. 3, EOPs are improved than EOPs of optical links of NRD $=0 \mathrm{ps} / \mathrm{nm}$ under the equal condition of launch power. That is, if the criterion value of EOP for the excellent reception performance is selected to $1 \mathrm{~dB}$ EOP, and launch power resulting below $1 \mathrm{~dB}$ EOP is defined as the allowable launch power, then the allowable launch power in optical links with NRD = $+10 \mathrm{ps} / \mathrm{nm}$ and $-10 \mathrm{ps} / \mathrm{nm}$, which are controlled by precompensation, for uniform RDPS distribution and descending-ascending RDPS configuration and for ascending-descending RDPS configuration, respectively, are increased by $1 \mathrm{dBm}$ than that obtained in optical links with $\mathrm{NRD}=0 \mathrm{ps} / \mathrm{nm}$, as shown in Fig. 4(a). Also, the allowable launch power in optical links with NRD = $10 \mathrm{ps} / \mathrm{nm}$ and $+10 \mathrm{ps} / \mathrm{nm}$, which are controlled by postcompensation, for uniform RDPS distribution and descending-ascending RDPS configuration and for ascending-descending RDPS configuration, respectively, are increased by $0.5 \sim 1 \mathrm{dBm}$ than that obtained in optical links with NRD $=0 \mathrm{ps} / \mathrm{nm}$, as shown in Fig. 4(b).

It is also confirmed that EOPs obtained in optical links of ascending-descending RDPS configuration and descending-ascending RDPS configuration are similar with that obtained in uniform RDPS distribution over all considered launch power. This result means that the effect of the exact RDPS distribution on system performance is not significant. Therefore, from the result of Fig. 4, implementation of the flexible optical links independent on RDPS value of each fiber span is possible.

The allowable launch powers depending on RDPS distribution are obtained from the results of Fig. 4, but, it is difficult to implement the flexible WDM transmission system, because only one optimal NRD value is used for designing optical links according to the result of Fig. 4. Fortunately, through the analysis the result of Fig. 3, it is also confirmed that the effective NRD inducing EOP below $1 \mathrm{~dB}$ at arbitrary launch power is distributed over wide range. That is, several effective NRD values for excellent receiving performance are presented at arbitrary launch power.

These NRD values resulting EOP below $1 \mathrm{~dB}$ are defined as the effective NRD range in this paper. Fig. 5 illustrates the effective NRD ranges depending on launch power of worst channel in optical links controlled by precompensation and postcompensation, respectively. Fig. 5 assists to flexibly design optical links for WDM transmis- 
sion, because it provides the relation between NRD range and launch power.

It is shown that the effective NRD ranges of ascendingdescending RDPS configuration and descendingascending RDPS configuration are similar with that of uniform RDPS distribution for relative low launch power, i.e., $-8 \sim 1 \mathrm{dBm}$. And, if wider NRD margin than $60 \mathrm{ps} / \mathrm{nm}$ $(0 \pm 30 \mathrm{ps} / \mathrm{nm})$ is required, launch power is selected to be $5 \sim 1 \mathrm{dBm}$ and $-6 \sim 1 \mathrm{dBm}$ in optical links controlled by precompensation and postcompensation, respectively, which are independence on RDPS distribution. It is also confirmed that the effective NRD range of uniform RDPS distribution is wider than that of ascending-descending RDPS configuration and descending-ascending RDPS configuration for relative high launch power $(5 \sim 6 \mathrm{dBm})$, but, in optical links controlled by precompenstion, the effective NRD range of ascending-descending RDPS configuration is wider than that of uniform RDPS distribution for launch power of $5 \sim 6 \mathrm{dBm}$.

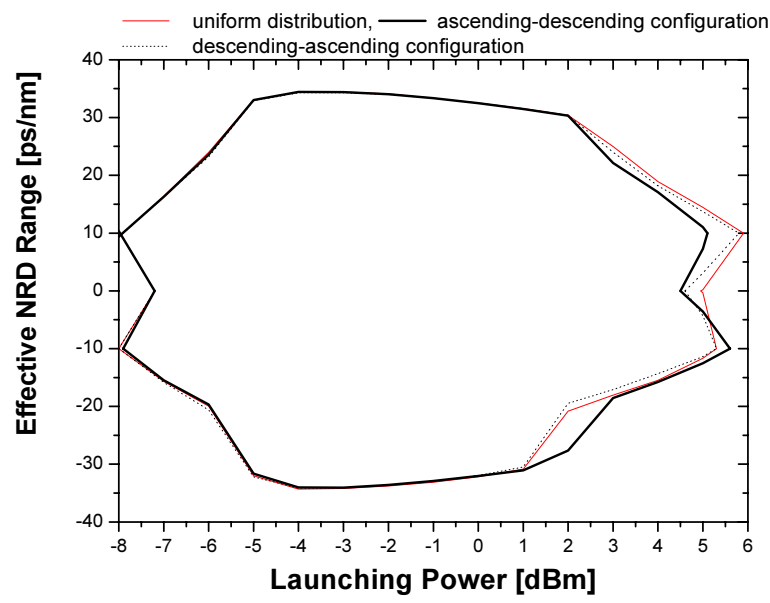

(a) precompensation

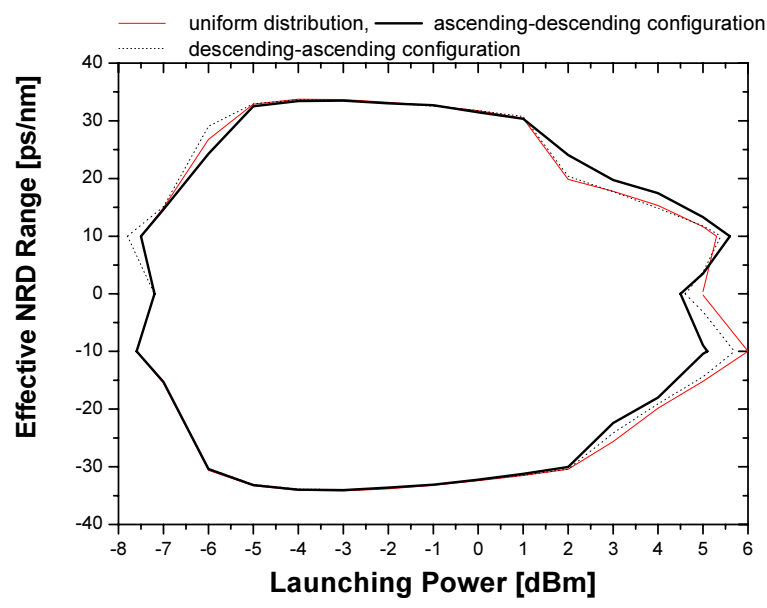

(b) postcompensation

Fig. 5. The effective NRD range for launch power.

\section{CONCLUSION}

This research discussed the possibility of implementing nonuniform RDPS distribution in optical links for WDM transmission. It was confirmed that the effect of nonuniform RDPS distribution on system performance is not significant, because the characteristics of EOP in ascending-descending RDPS configuration and descendingascending RDPS configuration is similar with that in uniform RDPS distribution for all considered launch power.

It was also confirmed that the optimal NRDs are obtained to $+10 \mathrm{ps} / \mathrm{nm}$ and $-10 \mathrm{ps} / \mathrm{nm}$ in optical link controlled by precompensation and postcompensation, respectively, for descending-ascending RDPS configurations, meanwhile, $-10 \mathrm{ps} / \mathrm{nm}$ and $+10 \mathrm{ps} / \mathrm{nm}$ in optical link controlled by precompensation and postcompensation, respectively, for ascending-descending RDPS configurations.

And, in this paper, the effective NRD range depending on launch power was provided for implementing flexible optical links. It was shown that the effective NRD ranges are independent on the exact RDPS distribution for relative low launch power, and, if launch power is selected to be $-5 \sim 1 \mathrm{dBm}$ and $-6 \sim 1 \mathrm{dBm}$ in optical links controlled by precompensation and postcompensation, respectively, wider NRD margin than $60 \mathrm{ps} / \mathrm{nm}$ is obtained.

\section{REFERENCES}

[1] G. P. Agrawal, Fiber-optic Communication Systems, 3rd ed. New York: John Wiley \& Sons, 2003

[2] R. Ramaswami, and K.N. Sivarajan, Optical Networks: a Practical Perspective, Morgan Kaufmann Publishers, Inc. California, 1998.

[3] S. Watanabe and M. Shirasaki, "Exact compensation for both chromatic dispersion and Kerr effect in a transmission fiber using optical phase conjugation", J. Lightwave Technol., Vol. 14, No. 3, pp. 243-248, 1996.

[4] Author of this paper, "Dispersion Managed Optical Transmission Links with Optimized Optical Phase Conjugator", International Journal of KIMICS, Vol. 7, No. 3, pp. 372 376, 2009.

[5] Author of this paper, "Asymmetricity of Optical Phase Conjugation in Optical Transmission Links with Dispersion Management", The Journal of Korea Information and Communications Society, Vol. 35, No. 8, pp. 801 809, 2010.

[6] Author of this paper, "Performance Improvement of WDM Channels using Inline Dispersion Management in Transmission Links with OPC Placed at Various Position", submitted in The Journal of Korea Navigation Institute

[7] G. P. Agrawal, Nonlinear Fiber Optics, 3rd ed. San Francisco: Academic Press, 2001.

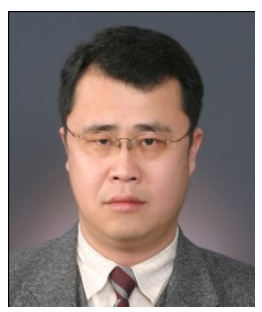

Seong-Real Lee (M'04) became a Member (M) of KIICE in 2004. He received the B.S., M.S. and Ph. D. degree in telecommunication and information eng. from Hankuk Aviation University, Korea in 1990, 1992 and 2002, respectively. He is currently an assistant professor at the Division of Marine Electronic and Communication Eng., Mokpo national Maritime University. His research interests include optical WDM systems, optical soliton systems and the optical nonlinear effects. 